\title{
Ultrasonido y Deshidratación Osmótica como Pretratamientos a la Liofilización de Melón (Cucumis melo L.)
}

\author{
Esmeralda S. Mosquera-Vivas ${ }^{(1) *}$, Alfredo A. Ayala-Aponte ${ }^{(1)}$ y Liliana Serna-Cock $^{(2)}$ \\ (1) Facultad de Ingeniería, Escuela de Ingeniería de Alimentos, Universidad del Valle, Calle 13 \#100-00, Campus \\ Universitario Meléndez, Santiago de Cali, Colombia. (e-mail: esmeralda.mosquera@correounivalle.edu.co; \\ alfredo.ayala@correounivalle.edu.co) \\ (2) Facultad de Ingeniería y Administración, Universidad Nacional de Colombia sede Palmira, Carrera 32 \#12-00 vía \\ Candelaria, Palmira, Colombia (e-mail: Iserna@unal.edu.co) \\ ${ }^{*}$ Autor a quien debe ser dirigida la correspondencia
}

Recibido Jul. 13, 2018; Aceptado Sep. 26, 2018; Versión final Nov. 17, 2018, Publicado Jun. 2019

\section{Resumen}

Se evaluó el efecto de dos pretratamientos, deshidratación osmótica (45Brix, 60 y 90min) y ondas de ultrasonido $(25 \mathrm{kHz}, 15$ y $30 \mathrm{~min})$, sobre la cinética de secado y parámetros fisicoquímicos de rodajas de melón (Cucumis melo L.). Rodajas pretratadas ( $4 \mathrm{~mm}$ de espesor y $20 \mathrm{~mm}$ de diámetro) se liofilizaron $\left(-40^{\circ} \mathrm{C}, 8 \mathrm{~Pa}\right)$; en los tiempos de liofilización 0,60,120, 180, 240 y 300 min, se midieron, contenido de humedad, difusividad, actividad de agua y porosidad. Como tratamiento control se utilizaron muestras liofilizadas no pretratadas. Para el ajuste de los datos experimentales se emplearon modelos matemáticos propuestos en la literatura. Se observó que la deshidratación osmótica aceleró el proceso de secado. El ultrasonido retardó considerablemente el proceso de secado y presentó menor difusividad y porosidad. Se alcanzaron valores de actividad de agua inferiores a 0.4 y coeficientes de difusividad en el rango de los reportados para diferentes alimentos.

\section{Ultrasound and Osmotic Dehydration as Pre-treatments to Melon (Cucumis melo L.) Drying by Freeze-drying}

\begin{abstract}
The effect of two pretreatments, osmotic dehydration (45ㅂ. Brix) (60 and 90min) and ultrasound waves (25kHz, 15 and $30 \mathrm{~min}$ ), on the drying kinetics and physicochemical parameters of melon slices (Cucumis melo) was evaluated. Pre-treated slices (thickness: $4 \mathrm{~mm}$, diameter: $20 \mathrm{~mm}$ ) were lyophilized $\left(-40^{\circ} \mathrm{C}, 8 \mathrm{~Pa}\right)$. In lyophilization times $0,60,120,180,240$ and 300 min, moisture content, diffusivity, water activity and porosity were measured. As a control treatment, lyophilized non-pretreated samples were used. Mathematical models proposed in the literature were used to adjust the experimental data. It was observed that osmotic dehydration accelerated the drying process. Ultrasound waves considerably delayed the drying process and presented lower values of diffusivity and porosity. Water activity values lower than 0.4 and diffusivity coefficients in the range of those reported for different foods were reached.
\end{abstract}

Keywords: drying kinetics; freeze drying; osmotic dehydration; ultrasound; Cucumis melo L. 


\section{INTRODUCCIÓN}

La liofilización se considera uno de los mejores métodos de secado que conserva en gran parte las propiedades organolépticas y nutricionales de los productos biológicos. Los alimentos liofilizados se caracterizan por su baja actividad de agua, alta porosidad (Harnkarnsujarit et al., 2016) y estado vítreo (Islam et al., 2015). El proceso consiste principalmente en la congelación del alimento y la posterior deshidratación por sublimación. Debido a la ausencia de agua líquida y las bajas temperaturas requeridas para la liofilización, la mayor parte del deterioro y las reacciones microbiológicas se retardan dando como resultado un producto final de alta calidad (Harnkarnsujarit et al., 2016). A pesar de sus ventajas su uso es limitado debido a los altos costos de energía relacionados con largos tiempos de secado. Para optimizar las condiciones en los procesos de deshidratación y disminuir los tiempos de proceso, se utilizan pretratamientos que permiten reducir el contenido de agua inicial del alimento, modifican los tejidos estructurales en la matriz sólida y generan efectos sobre la transferencia de masa (Arreola y Rosas, 2007; da Costa, Aguiar-Oliveira, y Maldonado, 2016; Fernandes, Gallão, y Rodrigues, 2008). Como pretratamientos a procesos de secado se han usado la deshidratación osmótica (DO) y ondas de ultrasonido (US).

La DO es una técnica ampliamente utilizada como pretratamiento a procesos de secado, para reducir pérdidas de calidad y disminuir el tiempo de proceso; La deshidratación osmótica incluye dos tipos de transferencia de masa: la difusión del agua del alimento a la solución y la difusión de solutos de la solución al alimento (Arreola y Rosas, 2007). La deshidratación se produce de forma natural, después de su inmersión en soluciones hipertónicas que presentan una alta presión osmótica y disminución de la actividad de agua como consecuencia de la diferencia de presiones (Amami et al., 2017). La diferencia de presión osmótica entre el alimento y la solución hipertónica, proporcionan la fuerza necesaria para el proceso de transferencia de masa, donde la estructura celular del alimento actúa como membrana semipermeable (da Costa et al., 2016).

Las US son recientemente utilizadas como pretratamiento a diversos procesos, que corresponden a una tecnología emergente que compite con las tecnologías convencionales en el procesamiento de alimentos (Islam et al., 2015). Las fuerzas involucradas en este mecanismo pueden ser más altas que la tensión superficial, lo que mantiene la humedad dentro de los capilares de la fruta creando canales microscópicos que podrían facilitar la eliminación de la humedad. En medio líquido, la sonicación causa cavitación, que consiste en la formación de burbujas en el líquido que pueden colapsar explosivamente y generar presión localizada (Fernandes, Linhares, y Rodrigues, 2008). Esta técnica se ha utilizado como pretratamiento al secado dado que provoca cambios en la estructura y acelera la transferencia de masa debido a los canales generados en la matriz del producto.

El secado es una operación compleja, que generalmente incluye fenómenos simultáneos de transferencia de calor y masa, las cinéticas de secado se han estudiado a través de diferentes formas fundamentales, fenomenológicas, cinemáticas y empíricas, cuando se puede suponer que las transferencias externas no son los fenómenos limitantes, la cinética de secado se revela principalmente por difusión del agua líquida contenida dentro de la matriz del alimento (Amami et al., 2017). Se han propuesto numerosos modelos matemáticos, empíricos y semiempíricos, para estimar las características de secado de los productos agrícolas. Estos modelos, también conocidos como modelos de capa delgada, permiten la predicción de la transferencia de masa durante la deshidratación y se aplican para simular curvas de secado (Sette, Salvatori y Schebor, 2016).

De otro lado, el melón (Cucumis melo L.) que pertenece a la familia de las Cucurbitáceas, es una fruta jugosa conocida por sus propiedades nutritivas y medicinales (Mallek-Ayadi et al., 2017). Debido a estas propiedades surge la importancia de mejorar su conservación y dar valor agregado como producto procesado. El objetivo de este estudio fue evaluar el efecto de la deshidratación osmótica y ondas de ultrasonido como pretratamientos al proceso de liofilización en rodajas de melón sobre las cinéticas de secado, contenido de humedad, porosidad, actividad de agua y difusividad efectiva, y evaluar modelos matemáticos para determinar el de mejor ajuste de los valores experimentales de humedad durante la cinética de secado.

\section{MATERIALES Y METODOS}

Se empleó melón (Cucumis melo L.) variedad Cantaloupe, para el control de la materia prima se utilizaron frutos entre 8 y $10^{\circ}$ Brix. Las frutas fueron adquiridas de un supermercado local y almacenadas en refrigeración a $4^{\circ} \mathrm{C}$ hasta su uso. Los frutos se lavaron con agua potable y se cortaron en láminas de $4 \mathrm{~mm}$ de espesor empleando un tajador manual y por medio de un sacabocados de acero inoxidable se obtuvieron rodajas de $20 \mathrm{~mm}$ de diámetro. Una vez obtenidas las rodajas de melón se sometieron inmediatamente a los pretratamientos de DO y US. 


\section{Pretratamiento con deshidratación osmótica (DO)}

Se preparó una solución hipertónica de $45^{\circ}$ Brix a $30 \pm 2 \stackrel{\circ}{ } \mathrm{C}$, usando sacarosa comercial como soluto osmótico por su amplio uso en procesos de DO y por su mayor peso molecular respecto a otros azucares, lo que provoca mayores pérdidas de agua (Azuara et al., 2002). La relación fruta - solución osmótica fue de 1:25, con el fin de asegurar una concentración constante de la solución durante el proceso osmótico (Ispir y Toğrul, 2009). Las muestras se sumergieron en la solución y se agitaron a $740 \mathrm{rpm}$ por medio de un agitador mecánico. Las muestras se retiraron de la solución osmótica en dos tiempos de proceso 60 (DO-60) y 90 min (DO-90) y se lavaron inmediatamente con agua destilada para retirar la solución osmótica remanente en la superficie de las muestras, posteriormente se secaron con papel absorbente para posterior liofilización.

\section{Pretratamiento con ultrasonido (US)}

Las muestras se sumergieron en agua destilada a $25^{\circ} \mathrm{C}$ en un recipiente de vidrio, la relación en peso fruta agua fue de 1:4 (Fernandes, Gallão, et al., 2008; Fernandes y Rodrigues, 2007). Estos recipientes se ubicaron en el equipo de ultrasonido (ELMA, USA) que consiste en un baño de acero inoxidable, con panel de control de frecuencia, temperatura y tiempo, las muestras se sometieron a ondas de frecuencia $25 \mathrm{kHz}$ en dos tiempos de proceso 15 (US-15) y 30 min (US-30). Posteriormente, las muestras pretratadas con US se colocaron sobre papel absorbente para retirar el exceso de agua y se sometieron al proceso de liofilización.

\section{Proceso de liofilización}

Después de los pretratamientos con DO y US las muestras se colocaron en un liofilizador de bandejas (LABCOMCO, USA) y se congelaron a $-40^{\circ} \mathrm{C}$. La deshidratación por sublimación se realizó a una presión de vacío de $8 \mathrm{~Pa}$. Para determinar las curvas de secado, las muestras se calentaron desde $-40^{\circ} \mathrm{C}$ hasta $20^{\circ} \mathrm{C}$ durante $300 \mathrm{~min}$ y se registraron las variaciones de humedad. Las variables de respuesta se midieron a diferentes tiempos de proceso (0,60,120,180, 240 y $300 \mathrm{~min})$, al retirar las muestras del liofilizador se empleó un desecador para evitar la ganancia de humedad. Los resultados de las muestras liofilizadas pretratadas en los dos tiempos de proceso osmótico 60 (DOLF-60) y 90 min (DOLF-90) y en los dos tiempos de US 15 (USLF15) y $30 \mathrm{~min}$ (USLF-30) se compararon con muestras frescas liofilizadas no pretratadas (LF).

\section{Determinación de propiedades fisicoquímicas}

El contenido de humedad $(\mathrm{CH})$ se determinó siguiendo el método AOAC 934,06 (AOAC, 1996). Se empleó una estufa de secado (Binder, E28, USA) a $70^{\circ} \mathrm{C}$ durante $24 \mathrm{~h}$ y una balanza analítica digital de $0.0001 \mathrm{~g}$ de precisión (Mettler-Toledo, AE100, Suiza). La actividad de agua (aw) se obtuvo mediante un equipo higrómetro (Aqualab 4TEV, USA). La porosidad $(\varepsilon)$ se calculó a partir de los valores de densidad real ( $\rho r$ ) y densidad aparente $(\rho a)$ empleando la Ecuación 1.

$$
\varepsilon=\frac{\rho_{\mathrm{r}}-\rho_{\mathrm{a}}}{\rho_{\mathrm{r}}} * 100
$$

La $\rho_{a}$ se determinó por el método de desplazamiento de volumen con un picnómetro a 25드 y la pr se calculó mediante la Ecuación 2 que relaciona el contenido de humedad y las densidades del agua y de los sólidos (Lewis, 1990). Donde $X_{w}$ es la fracción másica del agua.

$$
\rho_{\mathrm{r}}=\frac{1}{\frac{X_{w}}{997}+\frac{1-X_{w}}{1590}}
$$

\section{Modelación matemática}

Se emplearon los modelos matemáticos de Lewis (Ecuación 3), Henderson-Pabis (Ecuación 4) y Midilli (Ecuación 5) para describir las cinéticas de secado durante el proceso de liofilización, usados en el secado de alimentos y otros bioproductos, estos modelos son derivados del modelo difusional de la segunda ley de Fick para diferentes geometrías y condiciones del entorno (Puente-Díaz et al., 2013; Sette et al., 2016).

$$
\begin{aligned}
& M R=\exp (-k t) \\
& M R=a \exp (-k t) \\
& M R=a \exp \left(-k t^{n}\right)+b t
\end{aligned}
$$


Las constantes $\mathrm{k}, \mathrm{a}, \mathrm{b}, \mathrm{c}$ y $\mathrm{n}$ asociadas a los modelos se estimaron mediante una regresión no lineal, $\mathrm{k}$ representa parámetros cinéticos $\left(\mathrm{min}^{-1}\right)$; $\mathrm{a}, \mathrm{b}$, c y $\mathrm{n}$ son parámetros de correlación y t es el tiempo de secado (min). MR es la relación de humedad definida de acuerdo a la Ecuación 6 y simplificada en la ecuación 7.

$$
\begin{aligned}
& M R=\frac{X_{t}-X_{e}}{X_{o}-X_{e}} \\
& M R=\frac{X_{t}}{X_{o}}
\end{aligned}
$$

Donde $X_{\circ}$ representa el contenido de humedad en base seca (g agua/g m.s.) inicial del material, $X_{t}$ es el contenido de humedad después de un tiempo de secado y $X_{e}$ es la humedad en equilibrio, la cual no se tuvo en cuenta debido a que sus valores son muy pequeños comparados con $\mathrm{X}_{t}$ y $\mathrm{X}_{0}$.

Las regresiones para estimación de parámetros se realizaron utilizando el programa estadístico S.P.S.S 17.0. Como criterio de validez y comparación para evaluar el mejor ajuste de los modelos matemáticos a los datos experimentales de secado se utilizó el mayor valor del coeficiente de determinación $\left(R^{2}\right)$ más cercano a 1 y la raíz del error cuadrático medio (Ecuación 8) más cercano a cero (Sette et al., 2016).

$$
R M S E=\left[\frac{1}{N} \sum_{I=1}^{N}\left(M R_{p r e d}-M R_{\text {exp }}\right)^{2}\right]^{1 / 2}
$$

Donde $\mathrm{MR}_{\text {pred }} \mathrm{y} \mathrm{MR}_{\text {exp }}$ corresponden a la relación de humedad calculada con los datos predichos por los modelos matemáticos y con los datos experimentales respectivamente y $\mathrm{N}$ es el número de observaciones.

\section{Determinación de la difusividad efectiva}

La difusividad efectiva se calculó por medio de la solución analítica de la segunda ley de Fick (Ecuación 9) (Crank, 1975) para una geometría de lámina semi-infinita de espesor $L$ y considerando la difusión en dirección axial (Akgun y Doymaz, 2005; Ocoro y Ayala, 2012). Para tiempos de secado suficientemente largos, se consideró que el primer término en la expansión de la serie proporciona una buena estimación de la solución por lo tanto se simplificó en la Ecuación 10.

$$
\begin{aligned}
& M R=\frac{8}{\pi^{2}} \sum_{n=0}^{\infty} \frac{1}{(2 n+1)^{2}} \exp \left(-\frac{(2 n+1)^{2} \pi^{2} D_{\text {eff }} t}{4 L^{2}}\right) \\
& M R=\frac{8}{\pi^{2}} \exp \left(-\frac{\pi^{2} D_{e f f} t}{4 L^{2}}\right)
\end{aligned}
$$

Donde MR es la relación de humedad, $L$ es el espesor de la lámina $(m), t$ el tiempo de secado (s), $n$ es el número de términos de la sumatoria y $D_{\text {eff }}$ es la difusividad efectiva $\left(\mathrm{m}^{2} / \mathrm{s}\right)$.

\section{Análisis estadístico}

Los resultados experimentales se analizaron mediante un análisis de varianza (ANOVA) que determinó el efecto de las variables del proceso sobre las variables de respuesta. Todos los experimentos se desarrollaron por triplicado. Se determinó la diferencia de significancia entre promedios a un nivel de confianza del 95\%. El análisis de datos se realizó mediante el programa estadístico SPSS 17.0. Para identificar los tratamientos con diferencias significativas se aplicó la prueba de comparación de medias de Tukey.

\section{RESULTADOS Y DISCUSIÓN}

Los resultados y su análisis se presentan para cada una de las variables de respuesta cinéticas de secado, modelación matemática de las cinéticas de secado difusividad ( $\mathrm{d}_{\text {eff }}$ ) actividad de agua (aw) porosidad.

Cinéticas de secado

El CH de la fruta fresca fue de $10.397 \pm 0.332 \mathrm{~g}$ agua/g m.s, pero al aplicar los pretratamientos de DO y US previos a la liofilización las muestras alcanzaron valores de $2.763 \pm 0.126,1.551 \pm 0.097,15.608 \pm 0.443$ y $16.768 \pm 0.586 \mathrm{~g}$ agua/g m.s en DO-60, DO-90, US-15 y US-30 respectivamente. Es evidente la pérdida de agua con la DO, mientras que con US se incrementó. La pérdida y ganancia de agua se debe al gradiente de 
concentraciones entre la fruta y el medio líquido. Con la DO se produce un fenómeno de difusión simultánea, se favorece la transferencia de agua desde la fruta hacia la solución osmótica y la ganancia de sólidos desde la solución osmótica hacia la fruta (Amami et al., 2017; Fernandes, Linhares, et al., 2008). Mientras que con US se favorece la transferencia de agua desde el medio líquido de la sonicación hacia la fruta (Fernandes, Linhares, et al., 2008; Fernandes y Rodrigues, 2007) debido a la entrada de agua durante el tiempo de inmersión de 15 y 30 min. Este comportamiento es normal durante la aplicación del ultrasonido, debido a que estas ondas ultrasónicas pueden causar rápidas y sucesivas compresiones de la matriz sólida que podrían llenar los poros de la superficie del producto, sin embargo, se espera que durante el secado se extraiga esta cantidad de agua con mayor facilidad. Jambrak et al., (2007), encontraron resultados similares en la aplicación de US previo al secado de champiñones por aire caliente.

En la Fig.1 se presentan las curvas de secado de muestras de melón por liofilización. Los resultados mostraron que la pérdida de agua se incrementó con el tiempo de secado. De acuerdo con el análisis de varianza (ANOVA), el tipo de pretratamiento, el tiempo de DO y el tiempo de aplicación de US presentaron un efecto significativo $(\mathrm{p}<0,05)$ sobre el $\mathrm{CH}$ de la fruta. Se evidenció que las muestras con pretratamientos osmóticos (DOLF60 y DOLF90 min) presentaron menores tiempos de secado para alcanzar un nivel de humedad específico, siendo el tratamiento DOLF-90 el de menor tiempo. Para alcanzar un $\mathrm{CH}$ aproximado del $10 \%$ (b.s), los tratamientos DOLF-60 y DOLF-90 requieren respectivamente 179 y $174 \mathrm{~min}$, mientras que la muestra control LF y los tratados con US superan los $240 \mathrm{~min}$. Este resultado está asociado a la menor cantidad de agua en las muestras con DO al inicio del proceso de liofilización y a la mayor concentración de sólidos solubles de 17.2 y 31.0 Bix para DOLF-60 y DOLF-90 respectivamente. Resultados similares se han reportado en liofilización de pitahaya (Ayala et al., 2010) y frambuesa (Sette et al., 2016).

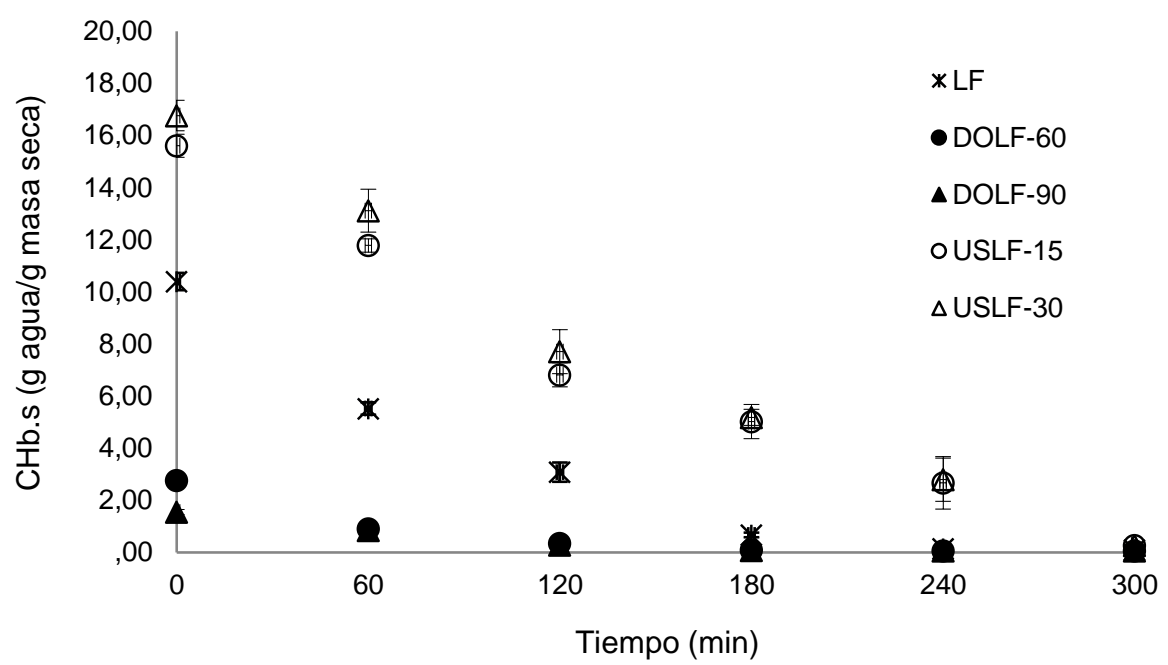

Fig. 1: Cinéticas de secado de rodajas de melón liofilizado

Al final del proceso (300 $\mathrm{min}$ ), el $\mathrm{CH}$ de las muestras fue de $0.095 \pm 0.009,0.066 \pm 0.003,0.063 \pm 0.001$, $0.274 \pm 0.048$ y $0.277 \pm 0.048 \mathrm{~g}$ agua $/ \mathrm{g}$ masa seca para LF, DOLF-60, DOLF-90, USLF-15 y USLF-30 respectivamente. Se corrobora que las muestras con pretratamiento osmótico (DO-60 y DO-90) presentaron los menores valores de humedad para un tiempo fijo, y al comparar estos dos tratamientos se observa que el tratamiento con mayor tiempo de deshidratación osmótica (DO-90) presentó el menor contenido de humedad final; este comportamiento está asociado a la alta concentración de azúcares o sólidos solubles en la fruta (31.0 ${ }^{\circ}$ Brix) y al menor contenido de humedad como se mencionó anteriormente. Similar comportamiento se ha reportado en piña osmodeshidratada y secada con aire caliente (Fernandes, Linhares, et al., 2008).

En las muestras pretratadas con US se presentaron los mayores contenidos de humedad, comportamiento no esperado, ya que se ha reportado en investigaciones que la combinación de US con otros métodos de secado ha sido positiva la disminución del tiempo de secado respecto a muestras sin US, como en el secado convectivo de mango (de Medeiros et al., 2016) y de manzana (Nowacka et al., 2012). Estos autores reportan que el US causa contracciones y expansiones rápidas del tejido celular de las frutas formando microcanales que facilitan la salida del agua intracelular de los productos.

Como explicación al comportamiento no esperado en el melón con aplicación previa de US en la liofilización, podría atribuirse al tiempo o a intensidad excesiva de las ondas sónicas provocando posibles cambios estructurales irreversibles impidiendo parcialmente la sublimación del agua propia del producto y la ganada durante el pretratamiento de sonicación. Estos cambios estructurales pueden estar asociados a la formación de innumerables microcanales en la matriz de la fruta, formándose en esta zona pequeños cristales de hielo 
durante la congelación lenta, no favoreciendo la difusión del vapor de agua durante la sublimación. Esta condición es desfavorable en el secado por liofilización, ya que a mayor tamaño de cristal de hielo más rápido es la deshidratación del producto (Wang et al., 2018). En investigaciones realizadas por otros autores se reportan micrografías SEM de muestras de melón en estado fresco, deshidratada osmóticamente y tratadas con US, los investigadores encontraron que las muestras tratadas con US presentaron cambios estructurales en las células de la fruta con menor tamaño y forma de agujas, mientras que en las frescas y deshidratadas osmóticamente no observaron estos cambios (Fernandes, Gallão, et al., 2008).

\section{Modelación matemática de las cinéticas de secado}

La Tabla 1 presenta los parámetros cinéticos estimados de los modelos de Lewis, Henderson-Pabis y Midilli. En general todos los modelos presentaron buen ajuste, pero el de Midilli fue el que mejor ajustó los valores experimentales de humedad durante el secado por presentar valores de R2 entre 0,988 y 1,0 y valores de RMSE entre 0,031 y 0,005 indicando que es un modelo apropiado para la predicción de la humedad en el secado de rodajas de melón por liofilización. Con el uso de modelos matemáticos se facilita la predicción de tiempos de procesamiento y de esta manera se contribuye a la optimización de la eficiencia del secado. El modelo de Midilli ha sido ampliamente utilizado en diferentes investigaciones de secado en diversas frutas y verduras (Nowacka et al., 2012; Puente-Díaz et al., 2013; Toğrul, 2005). El parámetro cinético k presentó los valores más altos en las muestras con pretratamientos osmóticos (DOLF-60 y DOLF-90) comparado con muestras sin pretratamiento y pretratadas con US. Este parámetro está fuertemente asociado con la mayor pérdida de agua y con la aceleración del secado a través del mecanismo de difusión desde el interior hacia la superficie del producto. La Fig.2 corrobora el buen ajuste de los valores de MR predichos con el modelo de Midilli frente a los experimentales en función del tiempo de secado para los diferentes tratamientos.

Tabla 1: Parámetros estimados con los modelos de Lewis, Henderson-Pabis y Midilli

\begin{tabular}{|c|c|c|c|c|c|c|c|c|c|c|c|c|c|}
\hline \multirow{2}{*}{ Modelos } & \multicolumn{4}{|c|}{ Lewis } & \multicolumn{4}{|c|}{ Henderson - Pabis } & \multicolumn{6}{c|}{ Midilli } \\
\cline { 2 - 15 } & $k$ & $R^{2}$ & $R M S E$ & $K$ & $a$ & $R^{2}$ & $R M S E$ & $k$ & $a$ & $n$ & $b$ & $R^{2}$ & $R M S E$ \\
\hline LF & 0,694 & 0,988 & 0,040 & 0,702 & 1,015 & 0,989 & 0,040 & 0,589 & 0,997 & 1,214 & $-0,002$ & 0,995 & 0,027 \\
\hline DOLF-60 & 1,080 & 0,998 & 0,011 & 1,079 & 0,998 & 0,998 & 0,011 & 1,109 & 1,000 & 0,999 & 0,003 & 1,000 & 0,007 \\
\hline DOLF-90 & 0,773 & 0,988 & 0,040 & 0,743 & 0,016 & 0,988 & 0,037 & 0,623 & 1,000 & 1,498 & 0,008 & 1,000 & 0,005 \\
\hline USLF-15 & 0,412 & 0,960 & 0,060 & 0,429 & 1,046 & 0,964 & 0,058 & 0,283 & 1,003 & 1,140 & $-0,022$ & 0,988 & 0,031 \\
\hline USLF-30 & 0,417 & 0,960 & 0,069 & 0,438 & 1,059 & 0,966 & 0,064 & 0,261 & 1,004 & 1,345 & $-0,010$ & 0,995 & 0,026 \\
\hline
\end{tabular}

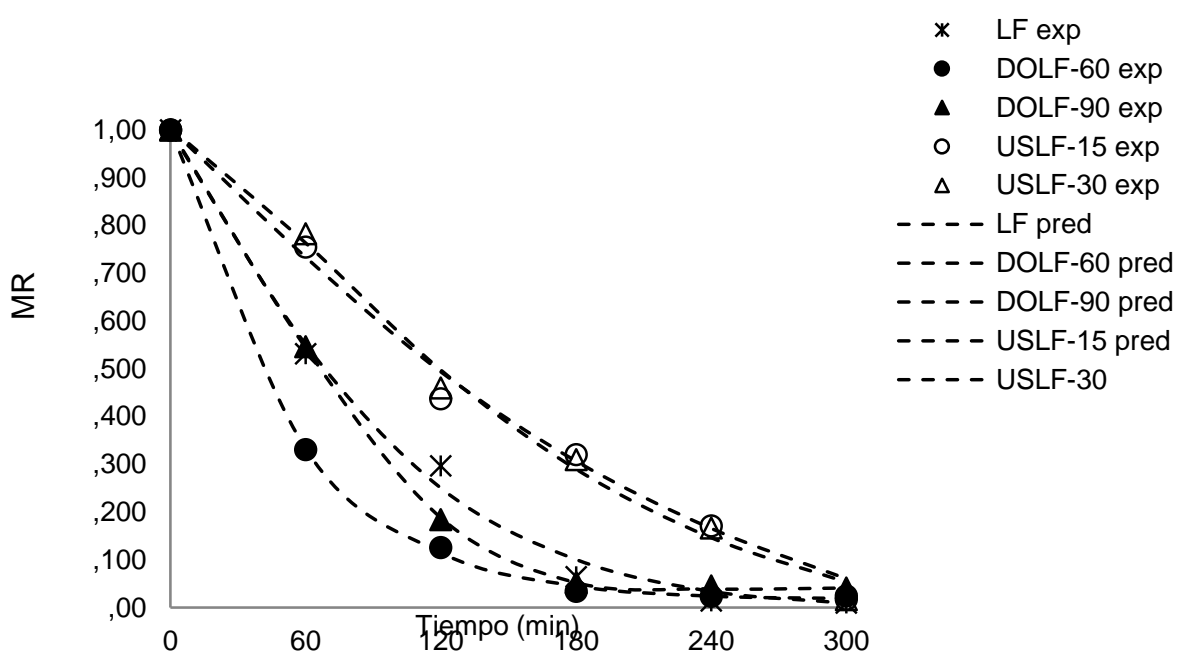

Fig. 2: Ajuste de los datos experimentales con el Modelo de Midilli

\section{Difusividad ( $\left.D_{\text {eff }}\right)$}

La Tabla 2 muestra los valores de difusividad efectiva (Deff) del agua en los diferentes tratamientos durante el proceso de liofilización. Los coeficientes presentaron una variación entre 1.904×10-9 y 8.000×10-10 m2/s, los cuales están en el rango de datos reportados en la literatura para muchas frutas como fresa, melón y piña (Amami et al., 2017; Fernandes, Gallão, et al., 2008; Fernandes, Linhares, et al., 2008). Según Marinos-Kouris y Maroulis (1995), la difusividad efectiva de los alimentos generalmente se encuentra en un rango de 10-13 a $10-6 \mathrm{~m}^{2} / \mathrm{s}$. 
Las muestras con US presentaron los menores valores de Deff, resultado atribuido a la dificultad de sublimar el hielo por los posibles cambios en la estructura de la matriz con la sonicación, como se mencionó anteriormente. Al comparar los tratamientos de DO con el tratamiento control, se presentó menor difusividad en las muestras tratadas osmóticamente, este resultado puede estar asociado a la mayor ganancia de azúcar que puede generar una barrera en la superficie de la fruta y ocasionar alta resistencia a la difusión de vapor de agua durante la sublimación. Comportamiento similar se observó en el secado de piña pretratada con DO seguido con aire caliente (Fernandes, Linhares, et al., 2008). El análisis estadístico presentó diferencia significativa

Tabla 2: Valores de coeficiente de difusión efectivo

(a,b Medias con letra común no son significativas)

\begin{tabular}{|c|c|}
\hline Tratamiento & $D_{\text {eff }}\left(\mathrm{m}^{2} / \mathrm{s}\right)$ \\
\hline LF $^{\mathrm{a}}$ & $1.904 \mathrm{E}-09$ \\
\hline DOLF-60 $^{\mathrm{a}}$ & $1.776 \mathrm{E}-09$ \\
\hline DOLF-90 $^{\mathrm{b}}$ & $1.440 \mathrm{E}-09$ \\
\hline USLF-15 $^{\mathrm{c}}$ & $8.000 \mathrm{E}-10$ \\
\hline USLF-30 $^{\mathrm{c}}$ & $8.000 \mathrm{E}-10$ \\
\hline
\end{tabular}

\section{Actividad de agua (aw)}

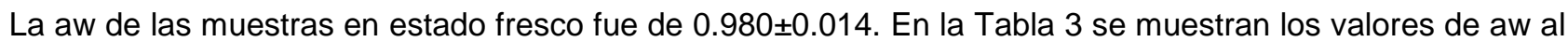
final del proceso de liofilización (300min). Se observa que todos los tratamientos mostraron valores inferiores de aw de 0.55 , lo cual indica que la fruta deshidratada es estable durante el almacenamiento contra el ataque de microorganismos por presentar valor inferior al agua critica de 0.6 (de Bruijn et al., 2016). De acuerdo a Bruijn et al. (2016), el contenido de humedad y la actividad del agua además de la temperatura, son los factores ambientales más importantes que afectan la tasa de reacciones de deterioro de los alimentos. Con niveles críticos de aw por encima de 0.85 se produce un deterioro indeseable de los productos. Por lo tanto, es importante reducir la actividad del agua a valores inferiores de 0,60 para que el producto sea estable y retenga los atributos de calidad de la materia prima. Sin embargo, valores más bajos de aw mantienen mayor estabilidad tanto microbiológica como en reacciones químicas de deterioro. Los tratamientos con DO y control (LF) alcanzaron los valores más bajos, inferiores a 0.4 , siendo consistentes con las pérdidas de humedad previamente analizadas. De acuerdo con Singh y Heldman (1993), valores de actividad de agua entre 0.2 y 0.4 garantizan la estabilidad del producto en el almacenamiento disminuyendo las reacciones de pardeamiento, oxidación hidrolítica, auto-oxidación y actividad enzimática. Tendencias similares se reportan en la osmoliofilización de frambuesas (Sette et al., 2016) y pitahaya (Ayala et al., 2010). El ANOVA presentó diferencia significativa entre pretratamientos $(p<0,05)$.

Tabla 3: Valores de aw en rodajas de melón liofilizado (a,b Medias con letra común no son significativas)

\begin{tabular}{|c|c|}
\hline Tratamiento & $a_{w}(300 \mathrm{~min})$ \\
\hline LF $^{\mathrm{a}}$ & $0,399 \pm 0.003$ \\
\hline DOLF-60 & $0,384 \pm 0.001$ \\
\hline DOLF-90 & $0.363 \pm 0.006$ \\
\hline USLF-15 & $0,482 \pm 0.004$ \\
\hline USLF-30 & $0,449 \pm 0.001$ \\
\hline
\end{tabular}

\section{Porosidad}

La Fig.3 muestra los cambios de la porosidad durante el proceso de liofilización. El valor de porosidad se incrementó en todos los tratamientos durante el tiempo de secado, asociado a la eliminación del agua, dejando espacios vacíos internos entre capilares y entre las estructuras celulares de la matriz de la fruta. Similar comportamiento se ha observado en el secado de zanahoria y manzana (Joardder et al., 2015). La microestructura de los alimentos es un parámetro clave para comprender las propiedades y la estabilidad de los alimentos (Harnkarnsujarit et al., 2016).

Al final del proceso (300 min) el ANOVA mostró diferencias significativas $(p<0.05)$ entre los pretratamientos con la muestra control, así como la comparación entre los tratamientos DO con los de US ( $p<0.05)$; sin embargo el tiempo de DO y del US no influyeron significativamente $(p>0.05)$ sobre la porosidad. Al final del secado se encontraron valores de porosidad de 77.703 $\pm 3.527,67.973 \pm 2.274,68.340 \pm 0.570,51.906 \pm 4.814$, $49.458 \pm 4.975 \%$ para LF, DOLF-60, DOLF-90, USLF-15 y USLF-30 respectivamente. Puede notarse que las muestras con pretratamiento osmótico y con US mostraron al final del secado valores inferiores respecto a la 
muestra control (LF). Este comportamiento es un indicador de algunos cambios estructurales en la matriz celular de la fruta por la aplicación de los pretratamientos. Con DO es posible que los poros externos se hayan saturado con solutos impidiendo la formación de poros internos (Torreggiani y Bertolo, 2001), comportamiento similar se observó en pitahaya amarilla (Ayala et al., 2010) y en calabaza (Mayor et al., 2011). Con respecto a las muestras tratadas con US, es posible que al formarse microcanales en la matriz de la fruta se hayan formado tamaños de cristales de agua más pequeños durante la congelación, y en consecuencia menor porosidad.

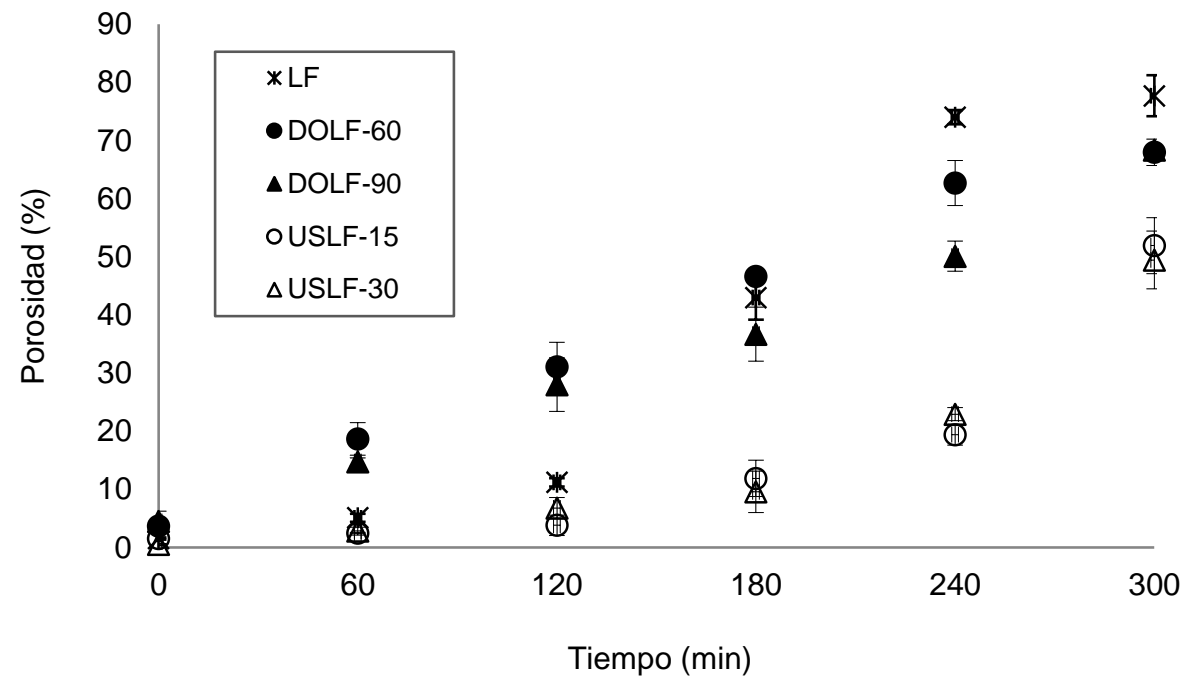

Fig.3: Porosidad en función del tiempo de rodajas de melón liofilizado

\section{CONCLUSIONES}

El uso de la deshidratación osmótica como pretratamiento al proceso de liofilización demostró que disminuye el tiempo de secado de rodajas de melón, a pesar de que la difusividad decrece a causa de la incorporación de azúcar. El uso de ondas de ultrasonido no favoreció el proceso de secado, el cual provocó mayor tiempo de liofilización respecto a las muestras tratadas con DO y a la muestra fresca sin pretratamiento osmótico, debido a posibles cambios de la estructura celular. Al final del secado las muestras control y los pretratamientos con DO y US presentaron estabilidad frente ataque de microorganismos por alcanzar valores de $a_{w}$ inferiores a 0.6. Las ondas de ultrasonido no favorecieron el incremento de la porosidad en el melón durante la liofilización. El modelo de Midilli presentó el mejor ajuste para la predicción de las cinéticas de secado de rodajas de melón por liofilización.

\section{REFERENCIAS}

Akgun, N.A. e I. Doymaz, Modelling of olive cake thin-layer drying process, doi: 10.1016/j.jfoodeng.2004.06.023, J. Food Eng., 68(4), 455-461 (2005)

Akgun, N.A., e I. Doymaz, Modelling of olive cake thin-layer drying process, doi: 10.1016/j.jfoodeng.2004.06.023, J. Food Eng., 68(4), 455-461 (2005)

Amami, E., W. Khezami y otros cinco autores, Effect of ultrasound-assisted osmotic dehydration pretreatment on the convective drying of strawberry, doi: 10.1016/j.ultsonch.2016.12.007, Ultrason. Sonochem., 36, 286-300 (2017)

AOAC International Method 934.06: Association of Official Analytical Chemists, Moisture in Dried Fruits, USA (1996)

Arreola, S.I. y M.E. Rosas, Aplicación de vacío en la deshidratación osmótica de higos (ficus carica), doi: 10.4067/S071807642007000200006, Inf. Tecnol., 18(2), 43-48 (2007)

Ayala, A.A., L. Serna y E. Mosquera, Liofilización de Pitahaya Amarilla (Selenicereus megalanthus), Rev. Facultad Quím. Farm., 17(2), 121-127 (2010)

Azuara, E., C.I. Beristain y G.F. Gutiérrez, Osmotic dehydration of apples by immersion in concentrated sucrose/maltodextrin solutions, doi: 10.1111/j.1745-4549.2002.tb00486.x, J. Food Process. Preserv., 26(4), 295-306 (2002)

Crank, J., The mathematics of diffusion, $2^{a}$ Ed., Oxford: Clarendon Press, London, England (1975)

da Costa, A.S., E. Aguiar-Oliveira y R.R. Maldonado, Optimization of osmotic dehydration of pear followed by conventional drying and their sensory quality, doi: 10.1016/j.Iwt.2016.04.062, Food Sci. Technol., 72, 407-415 (2016)

de Bruijn, J., F. Rivas y otros cinco autores, Effect of Vacuum Microwave Drying on the Quality and Storage Stability of Strawberries doi: 10.1111/jfpp.12691, J. Food Process. and Preserv., 40(5), 1104-1115 (2016) 
de Medeiros, R.A.B., Z.M.P. Barros y otros cuatro autores, Influence of dual-stage sugar substitution pretreatment on drying kinetics and quality parameters of mango, doi: 10.1016/j.Iwt.2015.11.049, Food Sci. Technol., 67, 167-173 (2016)

Fernandes, F.A.N. y S. Rodrigues, Ultrasound as pre-treatment for drying of fruits: Dehydration of banana, doi: 10.1016/j.ultsonch.2008.03.009, J. Food Eng., 82, 261-267 (2007)

Fernandes, F.A.N., F.E. Linhares y S. Rodrigues, Ultrasound as pre-treatment for drying of pineapple, doi: 10.1016/j.ultsonch.2008.03.009, Ultrason. Sonochem.,15(6), 1049-1054 (2008)

Fernandes, F.A.N., M.I. Gallão y S. Rodrigues, Effect of osmotic dehydration and ultrasound pre-treatment on cell structure: Melon dehydration, doi: 10.1016/j.Iwt.2007.05.007, Food Sci. Technol., 41(4), 604-610 (2008)

Harnkarnsujarit, N., K. Kawai, M. Watanabe y T. Suzuki, Effects of freezing on microstructure and rehydration properties of freeze-dried soybean curd, doi: 10.1016/j.jfoodeng.2016.03.014, J. Food Eng., 184, 10-20 (2016)

Islam, M.N., M. Zhang, H. Liu y C. Xinfeng, Effects of ultrasound on glass transition temperature of freeze-dried pear (Pyrus pyrifolia) using DMA thermal analysis, doi: 10.1016/j.fbp.2014.02.004, Food Bioprod. Process., 94, $229-238$ (2015)

Jambrak, A.R., T.J. Mason, L. Paniwnyk, y V. Lelas, Accelerated drying of button mushrooms, Brussels sprouts and cauliflower by applying power ultrasound and its rehydration properties, doi: 10.1016/j.jfoodeng.2006.10.009, J. Food Eng., 81(1), 88-97 (2007)

Joardder, M.U.H., C. Kumar, R.J. Brown y M.A. Karim, A micro-level investigation of the solid displacement method for porosity determination of dried food, doi: 10.1016/j.jfoodeng.2015.05.034, J. Food Eng., 166, 156-164 (2015)

Lewis, M., Physical properties of foods and food processing systems, 57-58, Ellis Horwood Limited, Reading, Kingdom United (1990)

Mallek-Ayadi, S., N. Bahloul y N. Kechaou, Characterization, phenolic compounds and functional properties of Cucumis melo L. peels, doi: 10.1016/j.foodchem.2016.10.117, Food Chem., 221, 1691-1697 (2017)

Marinos-Kouris, D. y Z.B. Maroulis, Transport Properties in the Drying of Solids, in Handbook of Industrial Drying, $2^{\mathrm{a}}$ Ed., McGill University, (1) 113-159, Quebec, Canada (1995)

Mayor, L., R. Moreira y A.M. Sereno, Shrinkage, density, porosity and shape changes during dehydration of pumpkin (Cucurbita pepo L.) fruits, doi: 10.1016/j.jfoodeng.2010.08.031, J. Food Eng., 103(1), 29-37 (2011)

Nowacka, M., A. Wiktor, M. Śledź, N. Jurek y D. Witrowa-Rajchert, Drying of ultrasound pretreated apple and its selected physical properties, doi: 10.1016/j.jfoodeng.2012.06.013, J. Food Eng., 113(3), 427-433 (2012)

Ocoro, M. y A. Ayala, Evaluación de la técnica de Ventana de Refractanciaß en el secado de puré de papaya (Carica papaya L.), Rev. Facultad Quím. Farm., 19(1), 72-74 (2012)

Puente-Díaz, L., K. Ah-Hen, A. Vega-Gálvez, R. Lemus-Mondaca y K. Di Scala, Combined Infrared-Convective Drying of Murta (Ugni molinae Turcz) Berries: Kinetic Modeling and Quality Assessment, doi: 10.1080/07373937.2012.736113, Drying Technol., 31(3), 329-338 (2013)

Sette, P., D. Salvatori y C. Schebor, Physical and mechanical properties of raspberries subjected to osmotic dehydration and further dehydration by air- and freeze-drying, doi: 10.1016/j.fbp.2016.06.018, Food Bioprod. Process, 100, 156-171 (2016)

Singh, R.P. y D.R. Heldman, Introduction to Food Engineering, 2a ed., San Diego: Academic Press, Inc., San Diego, USA (1993)

Toğrul, H., Simple modeling of infrared drying of fresh apple slices, doi: 10.1016/j.jfoodeng.2005.03.031, J. Food Eng., 71(3), 311-323 (2005)

Torreggiani, D. y G. Bertolo, Osmotic pre-treatments in fruit processing: Chemical, physical and structural effects, doi: 10.1016/S0260-8774(00)00210-7, J. Food Eng., 49(2-3), 247-253 (2001)

Wang, W., J. Yang y otros cuatro autores, Experimental and numerical investigations on freeze-drying of porous media with prebuilt porosity, doi: 10.1016/j.cplett.2018.04.008, Chem. Phys. Lett., 700, 80-87 (2018) 
